\title{
CX3CR1 regulates intestinal macrophage homeostasis, bacterial translocation, and colitogenic Th17 responses in mice
}

\author{
Oscar Medina-Contreras, ${ }^{1}$ Duke Geem, ${ }^{1}$ Oskar Laur, ${ }^{2}$ Ifor R. Williams, ${ }^{2}$ Sergio A. Lira, ${ }^{3}$ \\ Asma Nusrat, ${ }^{2}$ Charles A. Parkos, ${ }^{2}$ and Timothy L. Denning ${ }^{1,2}$ \\ 1Department of Pediatrics and 'Epithelial Pathobiology Research Unit, Department of Pathology and Laboratory Medicine, Emory University, \\ Atlanta, Georgia, USA. ${ }^{3}$ Immunology Institute, Mount Sinai School of Medicine, New York, New York, USA.
}

\begin{abstract}
The two most common forms of inflammatory bowel disease (IBD), Crohn's disease and ulcerative colitis, affect approximately 1 million people in the United States. Uncontrolled APC reactivity toward commensal bacteria is implicated in the pathogenesis of the disease. A number of functionally distinct APC populations exist in the mucosal lamina propria (LP) below the intestinal epithelium, but their relative contributions to inflammation remain unclear. Here, we demonstrate in mice important roles for the chemokine receptor CX3CR1 in maintaining LP macrophage populations, preventing translocation of commensal bacteria to mesenteric lymph nodes (mLNs), and limiting colitogenic Th17 responses. CX3CR1 was found to be expressed in resident $\mathrm{LP}$ macrophages (defined as $\mathrm{CD} 11 \mathrm{~b}^{+} \mathrm{F} 4 / \mathrm{80}^{+}$) but not DCs (defined as $\mathrm{CD} 11 \mathrm{c}^{+} \mathrm{CD} 103^{+}$). $\mathrm{LP}$ macrophage frequency and number were decreased in two strains of CX3CR1-knockout mice and in mice deficient in the CX3CR1 ligand CX3CL1. All these knockout strains displayed markedly increased translocation of commensal bacteria to mLNs. Additionally, the severity of DSS-induced colitis was dramatically enhanced in the knockout mice as compared with controls. Disease severity could be limited by either administration of neutralizing IL-17A antibodies or transfer of CX3CR1-sufficient macrophages. Our data thus suggest key roles for the CX3CR1/CX3CL1 axis in the intestinal mucosa; further clarification of CX3CR1 function will likely direct efforts toward therapeutic intervention for mucosal inflammatory disorders such as IBD.
\end{abstract}

\section{Introduction}

The vertebrate immune system is poised to potently respond against invading microbes and foreign molecules while enforcing tolerance toward self antigens (1). In the intestine, however, immune reactivity toward commensal flora must be restrained to prevent pathological inflammation, such as in Crohn's disease and ulcerative colitis $(2,3)$. There are numerous mechanical, physical, and immunological mechanisms that play crucial roles in maintaining intestinal homeostasis (4). Of the immunological parameters, components of the innate and adaptive immune systems cooperate to promote tolerance toward commensal flora while permitting reactivity to pathogens $(3,5)$.

Just beneath the epithelial barrier exist innate immune cells including APCs that direct appropriate B and T cell differentiation, and subsequent immunoglobulin and effector/regulatory $\mathrm{T}$ cell responses. Acquisition and presentation of food and bacterial antigens is believed to be carried out in large part by lamina propria (LP) DCs that migrate to the mesenteric lymph nodes (mLNs) in a CCR7-dependent manner (6), where they stimulate antigen-specific $\mathrm{B}$ and $\mathrm{T}$ cells to upregulate the gut-homing molecules $\alpha_{4} \beta_{7}$ and CCR9. mLN DCs also induce the differentiation of IgA-producing B cells and Foxp $3^{+}$regulatory T cells in the $\mathrm{mLN}$ via retinoic acid-dependent mechanisms (7-11). Thus, DCs in the $\mathrm{LP}$ and $\mathrm{mLN}$ play important roles in regulating intestinal $\mathrm{B}$ and $\mathrm{T}$ cell homing and differentiation.

Various different phenotypic markers have been used to identify and characterize subsets of LP DCs, and a number of conclusions

Conflict of interest: The authors have declared that no conflict of interest exists. Citation for this article: J Clin Invest. 2011;121(12):4787-4795. doi:10.1172/JCI59150. about the function of LP DCs have been made using these markers. In particular, the chemokine receptor CX3CR1 was proposed as a marker of LP DCs that extend transepithelial dendrites to sample luminal bacteria and mediate clearance of enteroinvasive pathogens (12). While CX3CR1 is expressed on many cell types and has long been appreciated as a marker for macrophages in the central nervous system (13), it has been frequently used as a marker for specific subsets of LP DCs (14). We and others have recently reported that CX3CR1 is not highly expressed on CD103+ LP DCs $(15,16)$ and that $\mathrm{CD} 103^{+}$LP cells, not CX3CR1 ${ }^{+}$cells, carry out classical DCmediated functions (16). There now appears to be a consensus that $\mathrm{CD} 11 \mathrm{c}^{+} \mathrm{CD} 103^{+} \mathrm{MHC} \mathrm{II}^{+}$cells are bona fide LP DCs (14); however, controversy remains over the lineage of CX3CR1-expressing LP cells and whether they are DCs or macrophages, with many groups referring to them as DCs, others as mononuclear phagocytes, and still others as macrophages (17). Since it has become increasingly appreciated that numerous subsets of DCs and macrophages exist within the intestinal LP $(14,18)$, we aimed to investigate the expression of CX3CR1 on these subsets, as well as the functional consequences of interfering with the CX3CR1/CX3CL1 axis. We examined LP DC and macrophage subsets in the small and large intestines of two independent lines of CX3CR1-deficient mice as well as CX3CL1deficient mice both in the steady state and during intestinal inflammation. In this report, we demonstrate an important role for the CX3CR1/CX3CL1 axis in regulating LP macrophage homeostasis and bacterial clearance and limiting colitogenic Th17 responses.

\section{Results}

Murine intestinal LP macrophage subsets preferentially express CX3CR1. In the intestine, LP DCs and macrophages play a central role in regulat- 
A

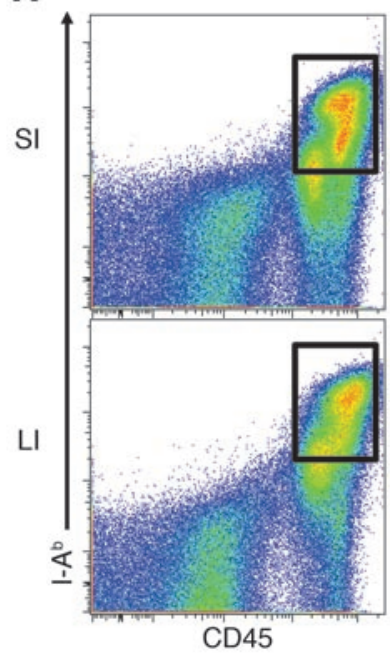

B
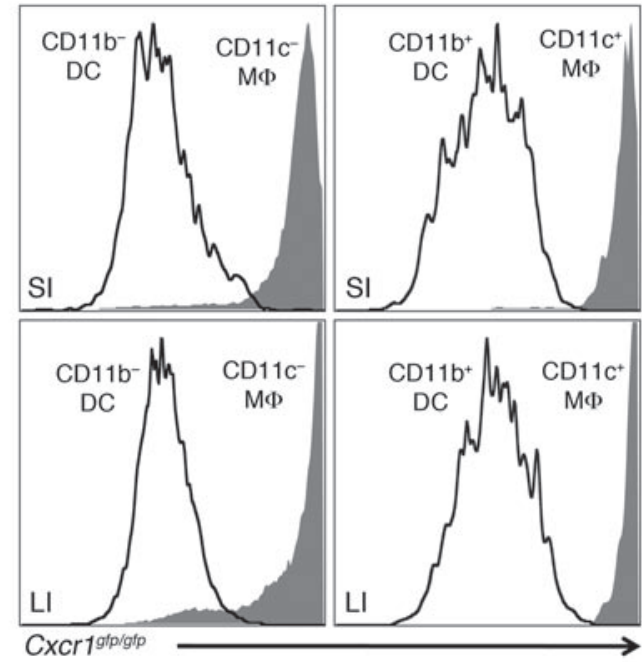

Gated on $\mathrm{CD}^{4} 5^{+} \mid-\mathrm{A}^{\mathrm{b}+}$

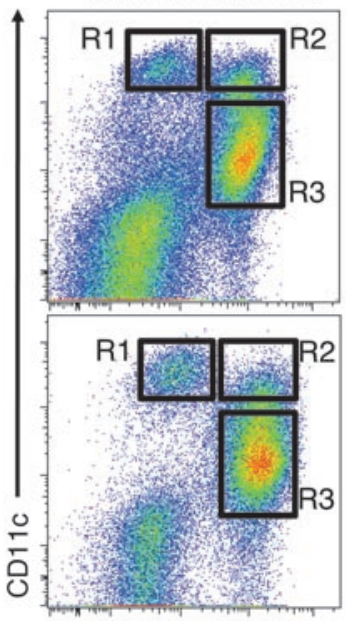

CD11b

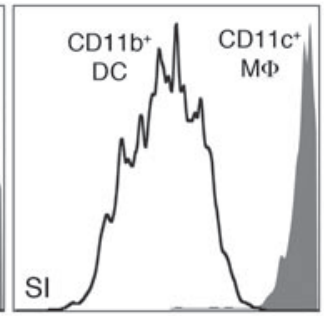

$11 \mathrm{c}^{+}$

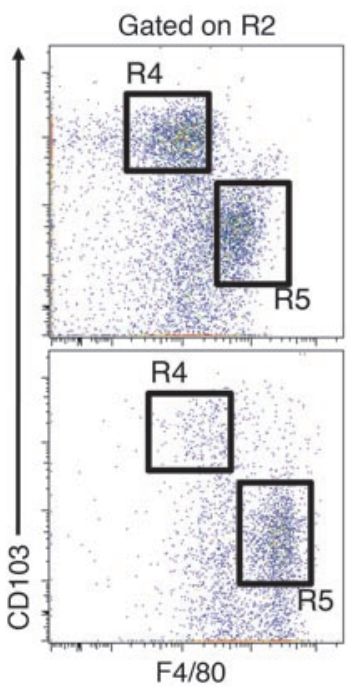

C

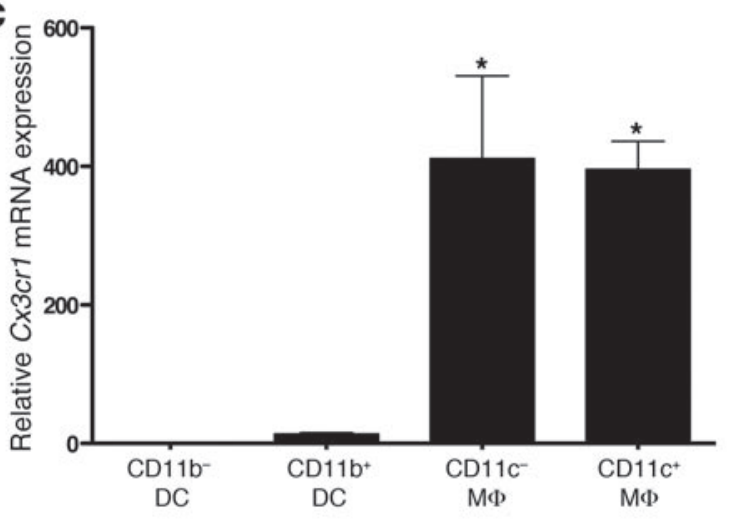

Figure 1

Murine intestinal LP macrophages express CX3CR1. (A) Flow cytometry of small (SI) and large (LI) intestine LP cells from C57BL/6 mice, stained for CD45, I-A , CD11b, CD11c, F4/80, and CD103. Populations were defined as R1, CD11 b- DCs; R3, CD11c- macrophages; R4, CD11b+ DCs; and R5, CD11 $\mathrm{c}^{+}$macrophages. (B) GFP expression in LP macrophages (M $\Phi$; solid histograms) and DCs (open histograms) in the small and large intestine of $\mathrm{C} \times 3 \mathrm{cr} 1$ gfp/gfp mice, assessed by flow cytometry and gated as in $\mathbf{A}$. (C) qRT-PCR for $C \times 3 c r 1$ mRNA expression in isolated intestinal LP APCs from $C \times 3 c r 1^{+/+}$mice. Data are representative of $3(\mathbf{B}$ and $\mathbf{C})$ or more than $3(\mathbf{A})$ independent experiments. Error bars represent SD. ${ }^{*} P<0.05$ versus respective controls.

ing innate and adaptive immune responses as well as inflammation. These various functions are carried out by different subsets of LP DCs and macrophages. Based on the expression of CD11b, CD11c, F4/80, and CD103, we have identified 4 populations of murine intestinal LP APCs, as defined as CD45 ${ }^{+} \mathrm{MHC} \mathrm{II}^{+}$cells (Figure $1 \mathrm{~A}$ and ref. 18):

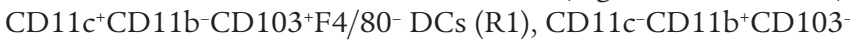
$\mathrm{F} 4 / 80^{+}$macrophages (R3), CD $11 \mathrm{c}^{+} \mathrm{CD} 11 \mathrm{~b}^{+} \mathrm{CD} 103^{+} \mathrm{F} 4 / 80^{-} \mathrm{DCs}(\mathrm{R} 4)$, and $\mathrm{CD} 11 \mathrm{c}^{+} \mathrm{CD} 11 \mathrm{~b}^{+} \mathrm{CD} 103^{-} \mathrm{F} 4 / 80^{+}$macrophages (R5). For simplicity, we refer to these populations as CD11b- DCs, CD $11 c^{-}$macrophages, $\mathrm{CD} 11 \mathrm{~b}^{+} \mathrm{DCs}$, and $\mathrm{CD} 11 \mathrm{c}^{+}$macrophages, respectively. It is reported that $\mathrm{CD} 11 \mathrm{~b}^{+} \mathrm{LP}$ DCs extend dendrites between neighboring IECs in order to sample luminal antigens in a CX3CR1-dependent manner $(12,19-21)$. However, CD11 b $\mathrm{b}^{+}$cells include a mixture of DC and macrophage subsets, and it is not clear which subsets express CX3CR1. Using Cx3cr1sf/gf reporter mice, which have $C \times 3 \mathrm{cr} 1$ replaced with gfp in both alleles, we analyzed the CX3CR1 expression on each of the LP macrophage and DC subpopulations. As shown in Figure 1B, we observed that GFP (CX3CR1) is expressed by CD11 ${ }^{-}$ and $\mathrm{CD} 11 \mathrm{c}^{+}$macrophages, but not by $\mathrm{CD} 11 \mathrm{~b}^{-}$or $\mathrm{CD} 11 \mathrm{~b}^{+} \mathrm{DCs}$, in both the small and large intestine. In order to rule out any possibility that GFP is not faithfully marking CX3CR1-expressing cells in this mouse model, we analyzed CX3CR1 expression in $\mathrm{C} \times 3 \mathrm{cr}^{+/+}$ C57BL/ 6 mice, using anti-CX3CR1 antibodies. Our results confirmed the GFP expression pattern, with specific CX3CR1 expression by CD11 $\mathrm{c}^{-}$and $\mathrm{CD} 11 \mathrm{c}^{+}$macrophages, but not $\mathrm{CD} 11 \mathrm{~b}^{-}$or

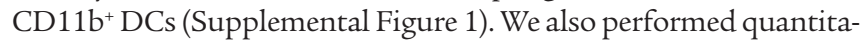
tive RT-PCR (qRT-PCR) on sorted CD $11 c^{-}$or CD $11 c^{+}$macrophages, as well as $\mathrm{CD}_{11 \mathrm{~b}^{-}}$or $\mathrm{CD} 11 \mathrm{~b}^{+} \mathrm{DCs}$. This further confirmed highly specific expression of CX3CR1 in both macrophages populations, but not in DC subsets (Figure 1C). 

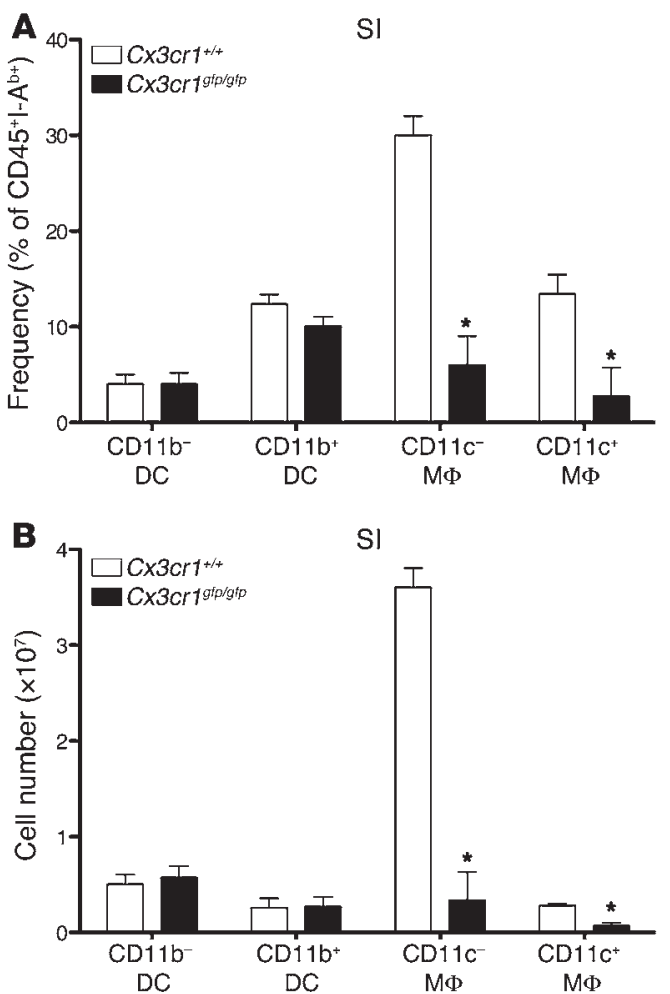

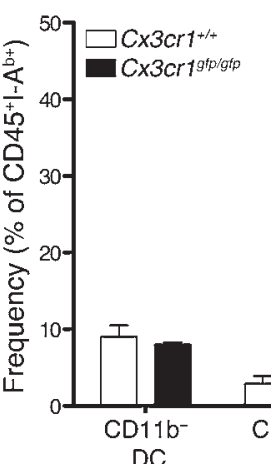

L

LI

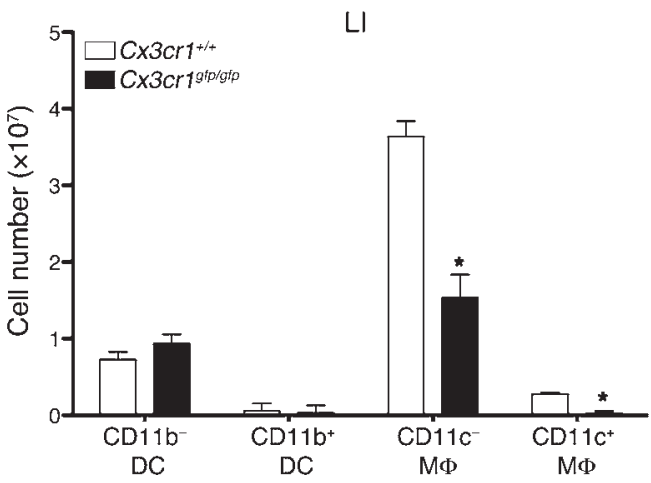

Figure 2

Cx3cr1gfp/gfp mice harbor a specific reduction in LP macrophages. (A) Percentage of CD45+MHC II+ macrophages and DCs in the small and large intestine of $C \times 3 c r 1$ gfp/gfp or $\mathrm{C} \times 3 \mathrm{Cr}^{+/+}$mice. (B) Total cellularity of CD45+MHC $\mathrm{II}^{+}$ macrophages and DCs in the small and large intestine of Cx3cr1 gfp/gfp or $\mathrm{C} \times 3 \mathrm{Cr}^{+1 /+}$ mice. (C) Percentage of bone marrow MDPs and blood monocytes of Cx3cr1 gfp/gfp or $\mathrm{C} \times 3 \mathrm{Cr}^{1+/+}$ mice. Data are representative of 3 (C) or more than 3 (A and $\mathbf{B}$ ) independent experiments. Error bars represent SEM. ${ }^{*} P<0.05$ versus respective controls.
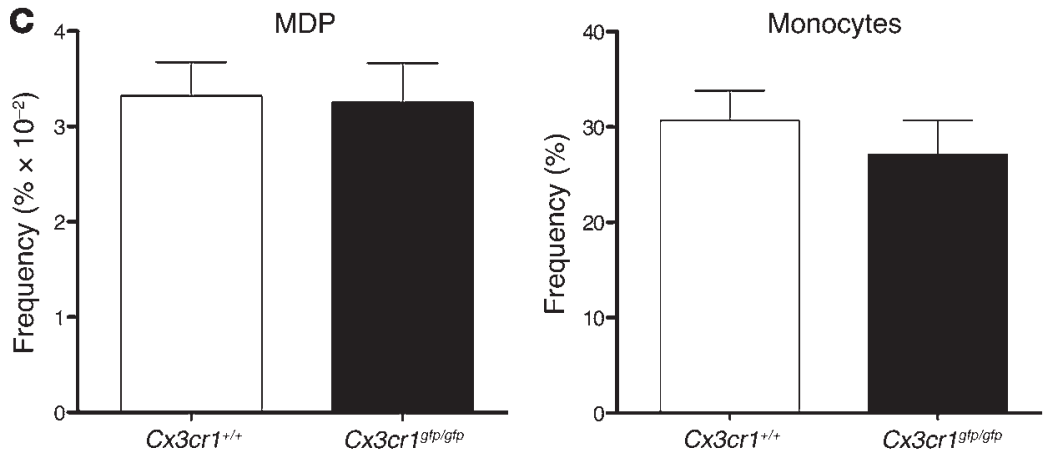

Reduction of LP macrophages in CX3CR1- and CX3CL1-deficient mice. Having observed specific expression of CX3CR1 by LP macrophages, we performed experiments to investigate the functional role of CX3CR1 in the intestine. Interestingly, phenotypic characterization of LP APCs in C $x 3 \mathrm{cr} 18 \mathrm{fP} / \mathrm{gf}$ mice revealed a significant reduction in the frequency of $\mathrm{CD} 11 \mathrm{c}^{-}$and $\mathrm{CD} 11 \mathrm{c}^{+}$macrophages in $\mathrm{C} \times 3 \mathrm{cr} 1 \mathrm{gfp} / \mathrm{gp}$ mice when compared with $\mathrm{C} \times 3 \mathrm{cr}^{+/+}$mice in both the small and large intestine (Figure 2A). This reduction was specific for LP macrophage subsets, as no significant differences were observed in the frequencies of $\mathrm{CD} 11 \mathrm{~b}^{-}$or $\mathrm{CD} 11 \mathrm{~b}^{+} \mathrm{LP}$ DCs between $\mathrm{C} \times 3 \mathrm{cr} 1 \mathrm{gfp} / \mathrm{ffp}$ mice and $\mathrm{C} x 3 \mathrm{cr} 1^{+/+}$mice. The total cell numbers of the $\mathrm{CD} 11 \mathrm{c}^{-}$and $\mathrm{CD} 11 \mathrm{c}^{+}$macrophage subsets were also significantly reduced in $C \times 3 c r 1 g f p / g f p$ mice, while DC subsets remained largely unaffected (Figure 2B). Since both LP macrophage subsets expressed high levels of GFP and were reduced in number, we wanted to verify that the observed reduction in LP macrophages was the result of CX3CR1 deficiency and not GFP-induced cytotoxicity. Therefore, we performed the same phenotypic characterization of LP DCs and macrophages in a different CX3CR1-deficient mouse line (22) that does not express GFP. Results using these $\mathrm{C} x 3 \mathrm{cr}^{-/-}$mice confirmed our observations with $C x 3 c r 1 g f / g f p$ mice, demonstrating a specific reduction in the frequency (Supplemental Figure $2 \mathrm{~A}$ ) and total cell

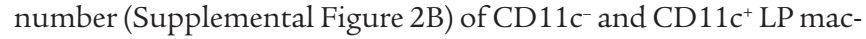

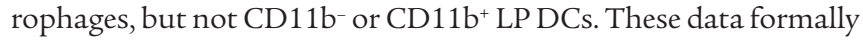
exclude GFP-induced cytotoxicity as the explanation for specific LP macrophage reduction in $\mathrm{Cx} 3 \mathrm{cr} 1 \mathrm{sp} / \mathrm{gfp}$ mice. Additionally, mice deficient in CX3CL1 (the ligand for CX3CR1) showed a reduction in LP macrophage subsets similar to that observed in CX3CR1-deficient mice (Supplemental Figure 3).

To evaluate whether the LP macrophage reduction in Cx3cr1sfp/gf mice is reflective of a general defect in monocyte/macrophage development or precursor accumulation, we analyzed the frequency of monocyte-DC common precursors (MDPs) in bone marrow as well as circulating blood monocytes (Figure 2C) and did not observe any significant difference in either of these populations. The significant LP macrophage reduction in CX3CR1-deficient mice was also not likely the result of increased cell death, since transfer of CD $45.2^{+} \mathrm{C} x 3 \mathrm{cr} 1^{+/+}$or CD $45.2^{+} \mathrm{C} \times 3 \mathrm{cr} 1 \mathrm{gfp} / \mathrm{gfp}$ bone mar- 

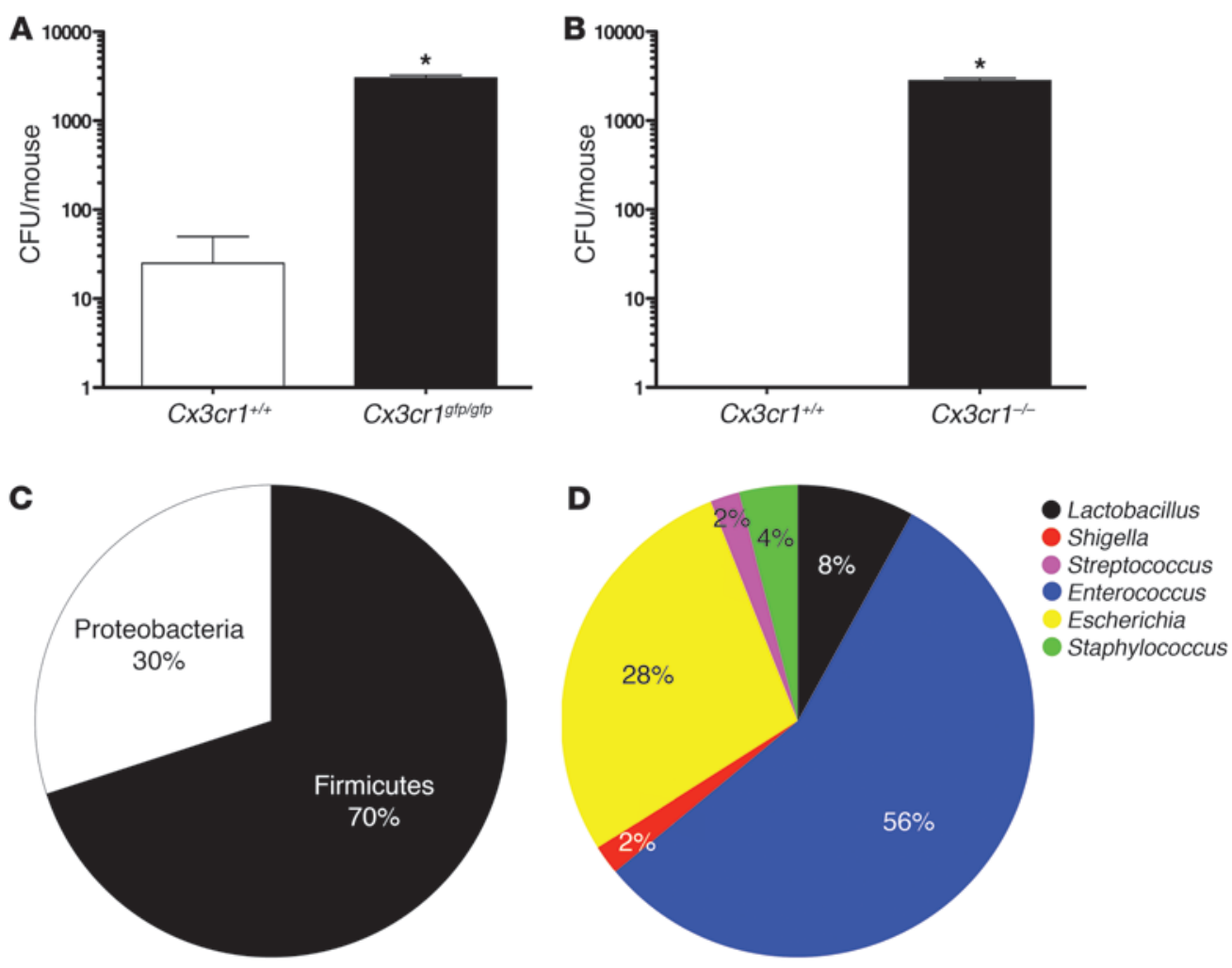

\section{Figure 3}

CX3CR1-deficient mice have increased bacterial translocation. CFU per mouse in the $\mathrm{mLNs}$ of Cx3cr1gfp/gfp (A) and $\mathrm{C} \times 3 \mathrm{cr} 1^{-1-}$ (B) mice. Relative abundance of phyla (C) and genera (D) among the translocated bacteria. Data are representative of 3 independent experiments, with 4 mice per group per experiment. ${ }^{*} P<0.05$ versus respective controls. row-derived macrophages (BMDMs) into CD $45.1^{+}$hosts resulted in equivalent numbers of $\mathrm{C} x 3 \mathrm{cr}^{+/+}$and $\mathrm{C} x 3 \mathrm{cr} 1^{g f p / g f p} \mathrm{BMDMs}$ in the spleens, but a significant reduction in $C x 3 \mathrm{cr} 1 \mathrm{gfp} / \mathrm{gfp}$ BMDMs in the intestines of recipient mice (data not shown). Taken together, our results demonstrate a specific reduction in LP macrophages in CX3CR1-deficient mice and suggest a role for the CX3CR1/ CX3CL1 axis in regulating LP macrophage migration and/or retention in the intestine. Interestingly, CX3CR1 deficiency also resulted in a significant reduction in macrophages in the lung and liver, but not the spleen (Supplemental Figure 4).

Increased bacterial translocation in CX3CR1- and CX3CL1-deficient mice. Given the phagocytic nature of LP macrophages (23) and their significant reduction in $C \times 3 \mathrm{cr} 1^{g f p} / g f p$ and $C \times 3 \mathrm{cr}^{-/-}$mice, we hypothesized that there may be a defect in the clearance of commensal bacteria that gain access to the lamina propria. To test this, we analyzed the $\mathrm{mLN}$ from these mice for signs of bacterial translocation. Total mLN cell suspensions cultured under aerobic conditions on LB blood agar plates revealed the presence of large numbers of bacteria in $\mathrm{C} \times 3 \mathrm{cr} 1^{1 \mathrm{fp} / \mathrm{gfp}}$ and $\mathrm{C} \times 3 \mathrm{cr}^{-/-}$mice (Figure 3, A and B) and in Cx3cl1-/- mice (Supplemental Figure 5A). In most experiments, bacteria could not be grown from mLNs of $\mathrm{C} \times 3 \mathrm{cr}^{+/+}$mice. The increased bacteria in the mLNs of CX3CR1deficient mice was not the result of defective intestinal barrier function, since there was no difference in the uptake of orally delivered, fluorescent $4-\mathrm{kDa}$ and $10-\mathrm{kDa}$ dextran into the serum of $\mathrm{C} x 3 \mathrm{Cr}^{+/+}$and CX3CR1-deficient mice (data not shown). Additionally, $\mathrm{C} x 3 \mathrm{cr}^{+/+}$and CX3CR1-deficient macrophages expressed similarly low to undetectable mRNA levels for molecules involved in forming adherens junctions (E-cadherin and $\beta$-catenin) and tight junctions (ZO-1, occludin, claudin-1; data not shown); thus, unlike what has been shown for DCs (24), macrophages may not play a role in directly maintaining intestinal epithelial barrier via tight junction protein expression.

To assess whether the bacteria present in the mLNs of CX3CR1deficient mice that grew under aerobic culture conditions were predominantly commensal or pathogenic bacteria, we isolated DNA from 50 individual colonies and performed PCR amplification using consensus 16S rDNA primers. Sequencing of PCR amplicons revealed that the translocated bacteria were predominantly commensal bacteria corresponding to the phylum Firmicutes (Figure 3C), with Enterococcus being the predominant genus (Figure 3D). Notably, a few sequences corresponded to Shigella; therefore, we cannot exclude the possibility that pathogenic bacteria can also gain access to the LP in the absence of CX3CR1. Overall, these results suggest that CX3CR1-expressing LP macrophages play a vital role in restricting translocation of commensal bacteria to the $\mathrm{mLN}$ during steady-state conditions.

Absence of CX3CR1 or CX3CL1 leads to enhanced intestinal inflammation. It has been shown that LP macrophages are important regulators of immune responses during intestinal inflammation $(25,26)$. Therefore, the specific reduction in LP macrophages in CX3CR1 deficiency prompted us to analyze how these mice respond to acute colitis induced by DSS. Beginning as early as 2 days following DSS administration, $C \times 3 c r 1^{g f p / g f p}$ and $C \times 3 \mathrm{cr}^{-/-}$mice exhibited significantly enhanced signs of intestinal disease when compared with $\mathrm{C} \times 3 \mathrm{crl}^{+/+}$mice, as defined by increases in overall disease activity index (DAI), a measure of weight loss, fecal blood, and soft stool/ diarrhea (Figure 4A). Histological analysis showed greater tissue damage and inflammatory infiltrate in DSS-treated C $\times 3 \mathrm{cr} 1^{g \mathrm{fp}} / \mathrm{gfp}$ and $\mathrm{C} \times 3 \mathrm{cr}^{-/-}$mice when compared with $\mathrm{C} x 3 \mathrm{cr}^{+/+}$mice (Figure 4B). Since mice lacking CX3CL1 also displayed a reduction in the frequency of both CD11 $c^{-}$and CD11 $c^{+}$LP macrophages (Supple- 


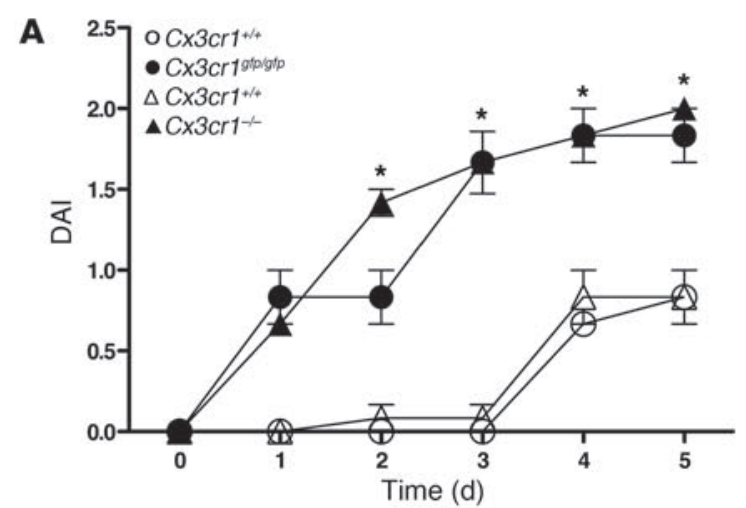

B

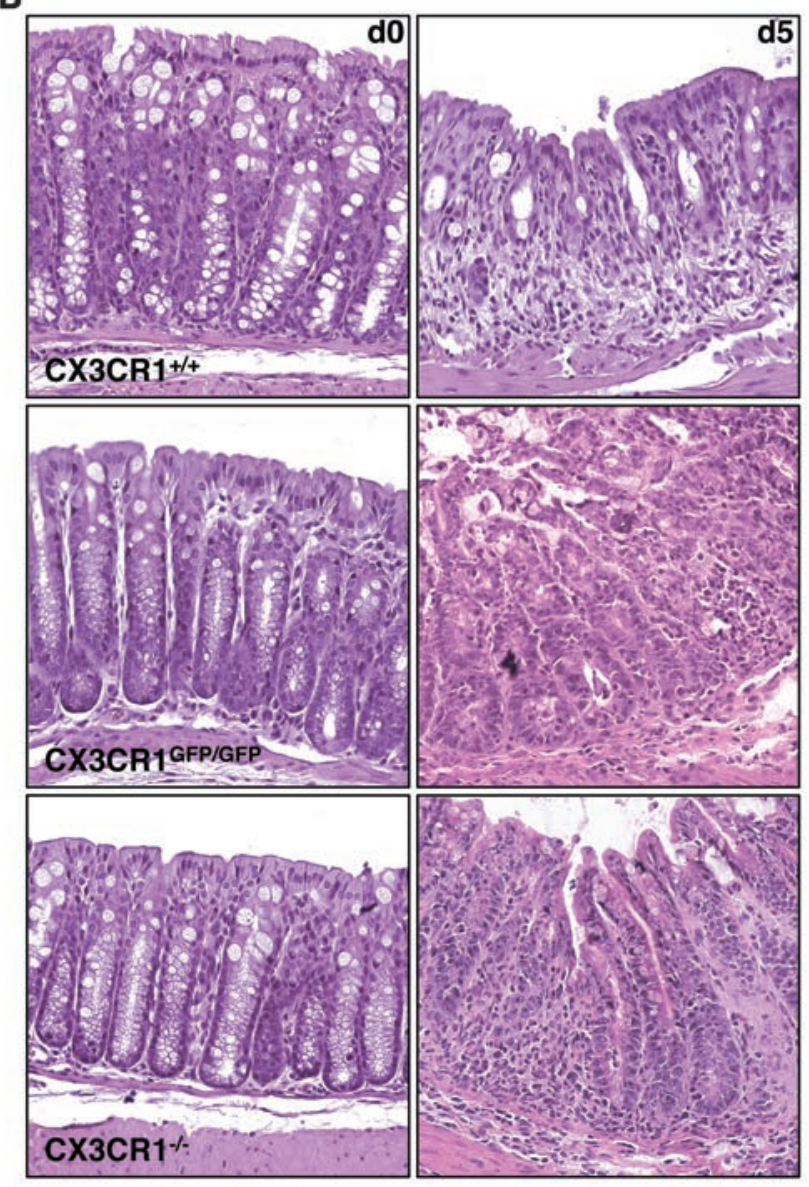

mental Figure 3) and increased bacterial translocation to the mLN (Supplemental Figure 5A), we investigated the response of these mice to DSS. Consistent with the increased sensitivity of $\mathrm{Cx} 3 \mathrm{cr} 1 \mathrm{gfp} / \mathrm{gfp}$ and $\mathrm{C} \times 3 \mathrm{cr}^{-/-}$mice, $\mathrm{C} \times 3 \mathrm{cll}^{-/-}$mice also displayed significantly enhanced sensitivity to DSS on days 3-5 (Supplemental Figure $5 \mathrm{~B})$. These data demonstrate that interfering with the CX3CR1/ CX3CL1 axis results in decreased recruitment and/or retention of macrophages to the intestinal LP, implying that there may be cells in the intestine that secrete CX3CL1 and recruit CX3CR1-expressing LP macrophages. To directly probe this possibility, we isolated RNA from intestinal epithelial cells (IECs) or total LP cells (nonIEC fraction) and performed qRT-PCR for Cx3cl1. Interestingly, we observed that both IECs and LP cells expressed robust levels of

\section{Figure 4}

Increased susceptibility to DSS-induced colitis in CX3CR1-deficient mice. (A) Severity of colitis in DSS-treated $\mathrm{C} \times 3 \mathrm{cr} 1^{\mathrm{gfp} / \mathrm{gfp}}$ and $\mathrm{C} \times 3 \mathrm{cr}^{-1-}$ mice or their respective $\mathrm{C} \times 3 \mathrm{Cr}^{+/+}$age- and vendor-matched control mice, as measured by stool consistency, presence of fecal blood, and weight loss. (B) Colon histology of DSS-treated Cx3cr1gfp/gfp, $\mathrm{C} \times 3 \mathrm{Cr}^{-1-}$, and $\mathrm{C} \times 3 \mathrm{Cr}^{+/+}$mice. Original magnification, $\times 400$. Data are representative of 3 independent experiments. Error bars represent SEM. ${ }^{*} P<0.05$.

Cx3cl1 mRNA (Supplemental Figure 5C). Collectively, these data suggest that regulation of CX3CL1 expression by IECs (27) or LP cells or the regulation of CX3CR1 expression by LP macrophages may have significant effects on macrophage homeostasis and intestinal inflammation.

Enhanced IL-17A-dependent colitis in CX3CR1-deficient mice. Since Th17 cells and Foxp $3^{+}$Tregs play important roles in regulating intestinal inflammation (3), we examined these $\mathrm{T}$ cell subsets in the steady state and during intestinal inflammation. Interestingly, under steady-state conditions, CX3CR1-deficient mice showed no significant differences in LP CD4 ${ }^{+}$Th17 cell or Foxp $3^{+}$Treg populations (Supplemental Figure 6) or IFN- $\gamma$-producing Th1 cells (data not shown). However, upon induction of intestinal inflammation, a 3.4-fold increase in colonic IL-17-producing $\mathrm{CD}^{+}$ $\mathrm{T}$ cells was observed in $\mathrm{C} \times 3 \mathrm{cr} \mathrm{l}_{\mathrm{gfp} / \mathrm{gfp}}$ mice, but not $\mathrm{C} \times 3 \mathrm{cr} \mathrm{1}^{+/+}$mice (Figure 5, A and B). Additionally, there was a trend toward reduced colonic Foxp $3^{+}$Tregs in C $x 3 \mathrm{cr} 1 \mathrm{gf} / \mathrm{gfp}$ mice during DSS treatment; however, this did not reach statistical significance (Figure 5, A and B). Since innate lymphoid cells are a recently appreciated source of IL-17 that contribute to the pathogenesis of intestinal inflammation (28), we investigated whether these cells were an additional source of IL-17A in colitic C $\times 3 \mathrm{cr} 1 \mathrm{ff} / \mathrm{gfp}$ mice. As shown in Supplemental Figure 7, CD3-ROR $\gamma \mathrm{t}^{+}$innate lymphoid cells were not an abundant source IL-17A in $C x 3 \mathrm{cr} 1 \mathrm{gP} / \mathrm{gfp}$ mice; thus, IL-17A is predominantly produced by $\mathrm{CD}^{+} \mathrm{LP} \mathrm{T}$ cells in colitic CX3CR1-deficient mice.

To investigate the possibility that the increased disease severity in CX3CR1-deficient mice is related to augmented IL-17A production, we induced acute colitis in $\mathrm{C} x 3 \mathrm{cr} 1 \mathrm{sp} / \mathrm{sp}$ and $\mathrm{C} \times 3 \mathrm{cr}^{+/+}$mice and injected neutralizing antibody against IL-17A or isotype control $\mathrm{IgG}_{1}$ at days 0,2 , and 4 . Figure $5 \mathrm{C}$ shows that while $\mathrm{IgG}_{1}$ treatment did not affect the severity of the disease in $C \times 3 \mathrm{cr} 1$ sfp/gfe and $\mathrm{C} \times 3 \mathrm{cr}^{+/+}$mice (when compared with Figure 4A), IL-17A neutralization significantly ameliorated the enhanced colitis symptoms in $\mathrm{C} \times 3 \mathrm{cr} 1^{g \mathrm{fp} / \mathrm{gfp}}$ mice, reducing them to levels comparable to those in $\mathrm{C} x 3 \mathrm{cr}^{1^{++}}$mice. This neutralization also significantly ameliorated colitis symptoms in $\mathrm{C} x 3 \mathrm{cr}^{-/-}$mice (Supplemental Figure 8). Importantly, IL-17A neutralization had little to no effect in modulating colitis in $\mathrm{C} x 3 \mathrm{cr} 1^{+/+}$mice (Figure 5C), thus emphasizing an important and unique role for IL-17A-producing $\mathrm{CD}^{+}$ $\mathrm{T}$ cells in enhanced colitis in CX3CR1-deficient mice. To further confirm that $\mathrm{CD}^{+} \mathrm{T}$ cells were involved in exacerbating colitis in CX3CR1-deficient mice, we treated Cx3cr1gfp/gfp mice with CD4depleting antibody and monitored disease activity. Complete depletion of $\mathrm{CD}^{+} \mathrm{T}$ cells in $\mathrm{Cx} 3 \mathrm{cr} \mathrm{l}^{g \mathrm{fp} / \mathrm{gfp}}$ mice (Supplemental Figure 9A) significantly reduced colonic inflammation as assessed by DAI (Supplemental Figure 9B). Therefore, $\mathrm{CD}^{+} \mathrm{T}$ cells are the major IL-17-producing cells contributing to the pathogenesis of colitis in CX3CR1-deficient mice. 


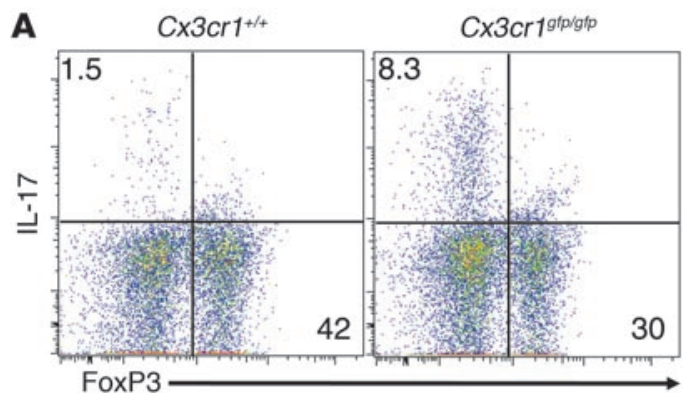$$
\text { B }
$$
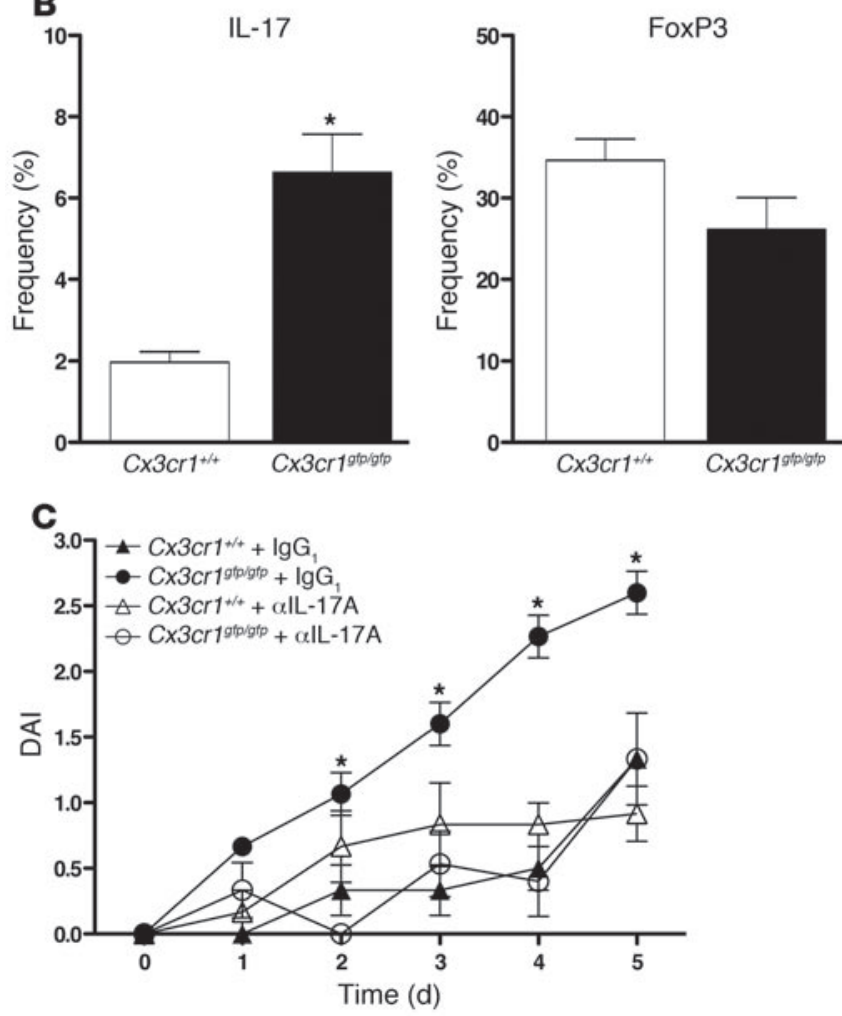

Transfer of $C \times 3 \mathrm{Cr}^{+/++}$macrophages suppresses colitis in CX3CR1-deficient mice. In order to probe the relationship between the reduction in LP macrophages and the increased susceptibility to DSS-induced colitis, we transferred $\mathrm{C} \times 3 \mathrm{cr} 1^{+/+} \mathrm{BMDMs}$ into $\mathrm{C} \times 3 \mathrm{cr} 1 \mathrm{ffp} / \mathrm{gfp}$ mice 1 day prior to and 1 day after initiating DSS treatment and followed clinical signs of intestinal inflammation. $\mathrm{C} \times 3 \mathrm{cr} 1^{+/+} \mathrm{BMDMs}$ exhibited a phenotype of CD45 hi, CD $11 b^{\text {hi }}, \mathrm{F} 4 / 80^{\text {hi }}$, MHC II ${ }^{\text {int }}$, $\mathrm{CX} 3 \mathrm{CR} 1^{+}, \mathrm{CD} 115^{+}, \mathrm{CD} 135^{\text {int }}, \mathrm{CD} 117^{-}$, indicating an intermediate phenotype between bone marrow precursors and tissue macrophages. These cells expressed low levels of CX3CR1 prior to transfer; however, CX3CR1 was upregulated after their differentiation in vivo (data not shown). This expression pattern is consistent with CX3CR1 expression increasing initially as MDPs differentiate into blood monocytes and further as blood monocytes differentiate into intestinal LP macrophages (data not shown).

Beginning at 3 days following DSS administration, C $\times 3 \mathrm{cr} 18 \mathrm{fp} / \mathrm{gfp}$ mice that received transfer of $C x 3 c r 1^{+/+}$BMDMs were significantly protected from colitis when compared with $C x 3 \mathrm{cr}$ 1 $^{g f} / g \mathrm{gp}$ mice not receiving transferred macrophages (Figure 6). The protection afforded to $\mathrm{C} \times 3 \mathrm{cr} 1_{\mathrm{gfp} / \mathrm{gfp}}$ mice by $\mathrm{C} \times 3 \mathrm{cr} 1^{+/+}$macrophages was similar

\section{Figure 5}

IL-17 responses contribute to enhanced colitis in $C \times 3 c r 1$ gfp/gfp mice. (A) Flow cytometry of intracellular Foxp3 or IL-17A by colonic CD4+ T cells from $\mathrm{C} \times 3 \mathrm{cr}$ 1 $^{\mathrm{gfp} / \mathrm{gfp}}$ or $\mathrm{C} \times 3 \mathrm{Cr} 1^{+/+}$mice. Numbers in outlined areas indicate percentage of cells in gate. (B) Percentage of $\mathrm{IL}-17 \mathrm{~A}^{+}$and FoxP3 $3^{+}$cells in the large intestine of $C \times 3 c r 1^{\text {gfp/gfp }}$ and $C \times 3 c r 1^{+/+}$mice. (C) Severity of colitis in DSS-treated $C \times 3 c r 1$ gfp/gfp or $C \times 3 \mathrm{Cr}^{+/+}$mice, in the presence of neutralizing IL-17A antibody or isotype control. Data are representative of $2(\mathbf{C})$ or $3(\mathbf{A}$ and $\mathbf{B})$ independent experiments. Error bars represent SEM. ${ }^{\star} P<0.05$ versus respective controls.

to that afforded by IL-17A neutralization. In contrast to $\mathrm{C} x 3 \mathrm{cr} 1^{+/+}$ BMDMs, transfer of $C x 3 c r 1 f f / g f p$ BMDMs did not provide any detectable protection to $C \times 3 \mathrm{cr} 1^{g f p / g f p}$ mice, even though $C \times 3 \mathrm{cr} 1^{g f p / g f p}$ macrophages were equally viable as $\mathrm{C} x 3 \mathrm{cr}^{+/+}$macrophages and capable of normal TNF production in response to LPS (data not shown). Additionally, transfer of either $C \times 3 \mathrm{cr} 1 \mathrm{gfp} / \mathrm{gfp}$ or $\mathrm{C} \times 3 \mathrm{cr} 1^{+/+}$ BMDMs into $\mathrm{C} x 3 \mathrm{cr}^{+/+}$mice (Supplemental Figure 10) showed no detectable effect on modulating DSS-induced colitis. Collectively, these data demonstrate that the expression of CX3CR1 on transferred macrophages is required for protecting CX3CR1deficient mice from increased sensitivity to DSS-induced colitis. Furthermore, homing of transferred macrophages to the intestine may be important for their ability to protect from colitis, since $\mathrm{C} \times 3 \mathrm{cr}^{+/+} \mathrm{BMDMs}$ were able to migrate to the intestine, as

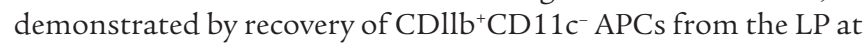
day 5 (Supplemental Figure 11).

\section{Discussion}

Together our experiments demonstrate that murine intestinal LP macrophages, but not LP DCs, express high levels of CX3CR1 and that deletion of CX3CR1 or CX3CL1 in vivo results in a specific and significant reduction in LP macrophages. The biological consequences of CX3CR1 deficiency included increased translocation of commensal bacteria to the $\mathrm{mLN}$, as well as enhanced Th17 responses and exacerbated disease during acute intestinal inflammation. Importantly, transfer of CX3CR1-sufficient macrophages or neutralization of IL-17A ameliorated the increased disease severity of CX3CR1-deficient mice. These results argue that CX3CR1 is not a marker for defining LP DCs, but rather a highly specific marker for LP macrophages and a critical component in maintaining LP macrophage homeostasis.

The specific expression of CX3CR1 by nearly all LP macrophages may offer insight into the findings that CX3CR1-expressing LP "DCs" are derived from monocyte precursors in the presence of GM-CSF (29-31). Since monocytes are well appreciated as the precursors of tissue-resident macrophages (32), it is most plausible that they are also the precursors of CX3CR1-expressing intestinal LP "macrophages." Additionally, the specific expression of CX3CR1 by LP macrophages suggests that bacterial clearance and luminal sampling via extension of dendrites, previously attributed to LP DCs $(12,19-21)$, may indeed be performed by LP macrophage subsets extending pseudopodia under certain circumstances. This concept is consistent with the avidly phagocytic nature of LP macrophages (23) and the recent finding that CX3CR1-expressing LP cells do not possess classical DC functions (16). Our results further suggest that the absence of transepithelial dendrites by "DCs" in Cx3crlgfp/gfp mice $(12,19,21)$ could be explained in part by the significant reduction in LP macrophages 


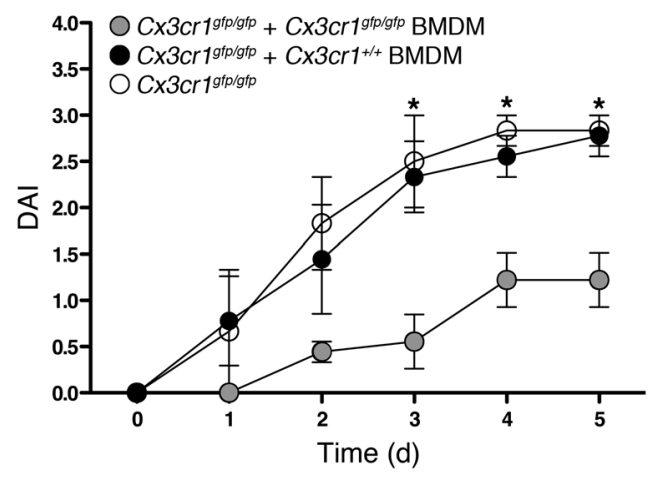

Figure 6

LP macrophage deficiency contributes to enhanced colitis in $C \times 3 c r 1$ gfp/gfp mice. Severity of colitis in DSS-treated $C \times 3 c r 1$ gfp/gfp mice after adoptive transfer of $C \times 3 c r 1^{\text {gfp/gfp }}$ or $\mathrm{C} \times 3 \mathrm{Cr} 1^{+/+}$BMDMs. Data are representative of 2 independent experiments. Error bars represent SEM. ${ }^{*} P<0.05$ versus respective controls.

and emphasize that data should be interpreted with caution when CX3CR1-deficient mice are used as a model system for investigating intestinal APC-dependent responses.

While the $\alpha_{4} \beta_{7}$ and CCR9 homing receptors are important for $\mathrm{T}$ and $\mathrm{B}$ cell migration to the intestine (33-35), far less is understood about the migration and/or retention of intestinal DCs and macrophages. The specific reduction in LP macrophages in CX3CR1- and CX3CL1-deficient mice demonstrates a clear link between this chemokine/receptor axis and the presence of these cells. Additional studies are required to clarify whether CX3CR1 and CX3CL1 are important for migration and/or retention of macrophages in the intestinal LP compartment in the steady state and during inflammation. CX3CR1 and CX3CL1 interactions may be important for maintaining antiinflammatory LP macrophage homeostasis in the steady state, while CCR2/CCL2 and/or other chemokine axes may regulate the accumulation of proinflammatory macrophages in the inflamed mucosa (36). This possibility is supported by recent data demonstrating the proinflammatory function of CX3CR $1^{\text {lo }}$ and CCR 2 hi $L P$ macrophages during intestinal inflammation $(36,37)$. Since the reduction in LP macrophages in CX3CR1- and CX3CL1-deficient mice is not complete, it is tempting to speculate that the remaining LP macrophages may be CX3CR $1{ }^{\text {lo }} \mathrm{CCR} 22^{\text {hi }}$ proinflammatory LP macrophages that contribute to enhanced intestinal inflammation. Our analyses of $C \times 3 c r 1^{g f p} / g f p$ reporter mice, however, revealed that these remaining LP macrophages still expressed high levels of GFP, indicating that they are not proinflammatory LP macrophages. Thus, remnant LP macrophages in CX3CR1-deficient mice may be phenotypically and functionally similar to steady-state antiinflammatory LP macrophages in wild-type mice, and it is their significant reduction in number that predisposes the host to bacterial translocation and exacerbated intestinal inflammation. It is important to note that CX3CR1 deficiency also affects macrophage abundance in the lung and liver; thus, the CX3CR1/CX3CL1 axis may play an important role in macrophage recruitment to several tissues in addition to the intestine.

In addition to the antiinflammatory functions of CX3CR1expressing LP macrophages, it has been reported that TNFproducing CX3CR1+ LP "DCs" contribute to the pathogenesis of colitis (30). These findings, seemingly contradictory to ours, may be explained by differences between transferred monocytes and resident LP macrophages in the duration of conditioning in situ. In particular, TNF-producing CX3CR1 ${ }^{+}$LP "DCs" (30) were generated following monocyte transfer in a relatively short time frame (2 weeks) compared with the long half-life of resident LP macrophages (16). Given that conditioning of LP macrophages by stromal cells may account in part for their antiinflammatory functions (23), our data suggest that resident CX3CR1 $1^{+}$LP macrophages are antiinflammatory, while $\mathrm{CX} 3 \mathrm{CR} 1^{+} \mathrm{LP}$ macrophages derived from recently infiltrating monocytes may have proinflammatory potential (30).

Recent evidence showed that CX3CR1 deficiency results in the reduction of IL-10 production by a minor subset of LP macrophages and abrogation of antigen-specific Foxp $3^{+}$Treg induction and oral tolerance, while total macrophage numbers remained unchanged (38). Interestingly, we observed that ablation of the

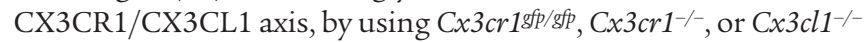
mice, resulted in a significant reduction in all $\mathrm{MHC} \mathrm{II}^{+} \mathrm{LP}$ macrophages subsets. We (15) and others (39) have shown that these major murine LP macrophage subsets produce copious amounts of IL-10, and thus a significant reduction in these cells may at least partially influence the observed biological effects of CX3CR1 deficiency on Treg induction and oral tolerance (38). Importantly, we did not observe a reduction in $\mathrm{CD}^{+}{ }^{+} \mathrm{Foxp} 3^{+}$Tregs in the LP of CX3CR1-deficient mice, indicating that CX3CR1-expressing LP macrophages may play a critical role in the induction (15) or maintenance (40) of Foxp $3^{+}$induced Tregs and a lesser role in maintenance of naturally existing Foxp $3^{+}$Tregs. Further experimentation is clearly required to delineate the role of CX3CR1 and specific macrophage and DC subsets in mediating intestinal tolerance to soluble oral antigens, as well as bacterial antigens.

Our findings that significant amounts of commensal bacteria translocated to the mLNs of CX3CR1- and CX3CL1-deficient mice are consistent with the phagocytic nature of LP macrophages (23). Since LP DC numbers are largely unaffected in the absence of CX3CR1 and CX3CL1, our data also imply that LP macrophages may be more important than LP DCs in phagocytosis and killing of commensal bacteria. In spite of commensal bacteria being present in the mLNs of CX3CR1-deficient mice, we did not observe any clinical signs of intestinal inflammation or innate immune cell activation in the mLN or intestinal LP; however, we did observe increases in B cells in the small and large intestine LP (data not shown). These data are consistent with increased bacteria in the mLN of JAM-A-deficient mice correlating with enhanced B cell follicles in the colonic LP (41).

Additionally, the dramatic increase in susceptibility to DSSinduced colitis in CX3CR1-deficient mice may be related to the reduction in phagocytic macrophages, thus creating an environment where commensal bacteria freely translocate following damage to the epithelial barrier. Interestingly, a recent report (42) proposed that CX3CR1 deficiency results in suppressed DSS-induced colitis in BALB/c mice. The reason for these discrepant observations are unclear but may be related to the strains of mice used or other unappreciated factors. We emphasize, however, that we have consistently observed enhanced DSS-induced colitis using two independent lines of CX3CR1-deficient mice as well as CX3CL1deficient mice (all on the C57BL/6 background).

Beyond their phagocytic capacity, LP macrophages may limit intestinal inflammation by promoting wound healing (43), 
dampening proinflammatory responses to TLR ligands, and inhibiting Th17 responses (15). We have previously demonstrated that LP macrophages can inhibit the induction of Th17 cells by CD $11 b^{+}$LP DCs in vitro (15) and speculate that the enhanced Th17 responses during intestinal inflammation in CX3CR1-deficient mice may be the result of a loss of macrophage-mediated regulation of $\mathrm{CD} 11 \mathrm{~b}^{+}$LP DCs. Further, the enhanced sensitivity of CX3CR1-deficient mice to colitis could be entirely reversed by neutralization of IL-17 or transfer of CX3CR1-expressing macrophages. While there are pathogenic and protective roles for IL-17 during homeostasis and intestinal inflammation (44-46), our data highlight an important, colitogenic role for IL-17-producing $\mathrm{CD}^{+} \mathrm{T}$ cells in the absence of CX3CR1. Collectively, our data support a role for the CX3CR1/CX3CL1 axis in regulating LP macrophage homeostasis, bacterial translocation, and IL-17 responses during intestinal inflammation. Further clarification of the functions of CX3CR1 in the steady state and during acute and chronic (21) colitis will likely contribute to a better understanding of mucosal immune regulation and direct efforts aimed at therapeutic intervention for mucosal inflammatory disorders, such as Crohn's disease and ulcerative colitis.

\section{Methods}

Mice. Six- to 8-week-old male Cx3cr1gp/gfp mice and age-matched $\mathrm{C} \times 3 \mathrm{crr}^{+/+}$ mice were purchased from The Jackson Laboratory. Six- to 8-week-old male $\mathrm{C} x 3 \mathrm{cr}^{-/-}$mice and age-matched $\mathrm{C} x 3 \mathrm{cr}^{+/+}$mice were purchased from Taconic. $\mathrm{C} x 3 \mathrm{Cl1}^{-/-}$mice were provided by Sergio Lira (Mount Sinai School of Medicine, New York, New York, USA) (47). Mice were maintained under specific pathogen-free conditions at Emory Division of Animal Resources facilities. All animal protocols were reviewed and approved by the Institutional Animal Care and Use Committee of Emory University.

Antibodies and reagents. The following antibodies were used: CD45-PerCP (30F11; BD), CD103-PE (M290; BD), CD4-PerCP (RM4-5; BD), IL-17A (TC11-18H10; BD), CD11c-allophycocyanin (N418; eBioscience), MHC II (I-A ${ }^{\mathrm{b}}$-Alexa Fluor 700 (M5/114.15.2; eBioscience), CD11b-eFluor 450 (M1/70; eBioscience), F4/80-PE and F4/80-PE-Cy7 (BM8; eBioscience), TCR $\beta$-allophycocyanin (H57-597; eBioscience), and FoxP3-eFluor 450 (FJK-16s; eBioscience). Anti-IL-17A (17F3) and mouse IgG 1 (MOPC-21) were purchased from Bio-X-Cell. Dead cells were identified using the fixable Aqua Dead Cell Staining Kit (Invitrogen). Recombinant murine M-CSF was from Peprotech. Dextran sulfate sodium (molecular mass, 40-50 kDa) was from USB Corp.

Isolation of LP cells. Isolation of LP cells was performed as previously described with modifications (15). Briefly, small intestines were removed and carefully cleaned of their mesentery, and Peyer's patches were excised. Small and large intestine were opened longitudinally, washed of fecal contents, cut into pieces $0.5 \mathrm{~cm}$ in length, and subjected to two sequential 20 -minute incubations in HBSS with $5 \%$ FCS and $2 \mathrm{mM}$ EDTA at $37^{\circ} \mathrm{C}$ with agitation to remove epithelial cells. After each incubation step, media containing epithelial cells and debris was discarded. The remaining tissue was minced and incubated for 20 minutes in HBSS with $5 \%$ FCS, $1 \mathrm{mg} / \mathrm{ml}$ collagenase IV (Sigma-Aldrich), and $40 \mathrm{U} / \mathrm{ml}$ DNase I (Roche) at $37^{\circ} \mathrm{C}$ in agitation. Cell suspensions were collected and passed through a $100-\mu \mathrm{m}$ strainer and pelleted by centrifugation at $300 \mathrm{~g}$.

Flow cytometry. Isolated LP cells were resuspended in PBS containing 5\% FBS. Live cells were identified using an Aqua Dead Cell Staining Kit accordingly to the manufacturer's instructions, and Fc receptors were blocked with the antibody anti-Fc $\gamma$ RIII/II (2.4G2) for 15 minutes at $4{ }^{\circ} \mathrm{C}$. After incubation the cells were stained at $4{ }^{\circ} \mathrm{C}$ for 30 minutes with labeled antibodies. Samples were then washed 2 times in PBS con- taining 5\% FBS and analyzed immediately, or intracellular staining was performed using a FoxP3 fixation/permeabilization kit (eBioscience) or Cytofix/Cytoperm kit with GolgiPlug (BD). Flow cytometric analysis was performed on a LSR II (BD).

Real-time PCR. Total RNA was isolated from purified LP macrophages and DCs using the QIAGEN RNeasy Mini Kit, according to the manufacturer's protocol, with on-column DNase digestion using the RNaseFree DNase set. cDNA was generated using the Superscript First-Strand Synthesis System for RT-PCR and random hexamer primers (Invitrogen), according to the manufacturer's protocol. cDNA was used as a template for qRT-PCR using SYBR Green Master Mix (Bio-Rad), and primers for Cx3cr1 (F, AGGACACAGCCAGACAAG; R, TCAGGGGAGAAAGCAAG) and Gapdh (F, TGGCAAAGTGGAGATTGTTGCC; R, AAGATGGTGATGGGCTTCCCG). PCR and analysis were performed using a MyiQ iCycler (Bio-Rad), and gene expression was calculated relative to Gapdh.

DSS-induced colitis. Mice were provided 3\% (wt/vol) DSS dissolved in the drinking water for 5 days. Daily clinical assessment of DSS-treated animals included measurement of a validated clinical DAI ranging from 0 to 4 (41), which was calculated using the following parameters: stool consistency, presence or absence of fecal blood (Hemoccult, Beckman Coulter), and weight loss. Mice were killed at day 5, and colons were assessed for histology.

Blocking of cytokine effect in vivo. CX3CR1-deficient (Cx3cr1sp/sfp or Cx3cr1-/-) or $\mathrm{C} x 3 \mathrm{cr}^{+/+}$mice were treated with DSS as described above. Mice were i.p. injected with $200 \mu \mathrm{g}$ of neutralizing IL-17A or isotype control antibodies at days 0,2 , and 4 . Colitis was assessed as described above.

Bone marrow adoptive transfer. Total bone marrow cells from Cx3cr1 $1 \mathrm{fp} / \mathrm{gfp}$ or $\mathrm{C} \times 3 \mathrm{CrI}^{+/+}$mice were cultured in the presence of $10 \mathrm{ng} / \mathrm{ml} \mathrm{M}$-CSF. Medium was replaced every 2 days, and at day 7 adherent macrophages were harvested. BMDMs $\left(1 \times 10^{6}\right)$ were adoptively transferred (tail vein injection) into

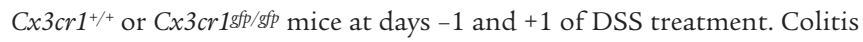
was assessed as described above.

Bacterial culture. Total $\mathrm{mLN}$ cell suspension was cultured under aerobic conditions in LB blood agar plates for 24 hours at $37^{\circ} \mathrm{C}$. Total DNA was directly isolated from individual colonies and sequenced for $16 \mathrm{~S}$ rDNA gene using the following primers: F, AGAGTTTGATCACTGGCTCAG; R, CTGCTGCCTCCCGTAGGAGT. Homology alignment was performed using NCBI BLAST software.

Statistics. Statistical analysis was performed with Prism software (GraphPad Software) using an unpaired 2-tailed Student's $t$ test. Error bars represent SD or SEM as indicated. $P$ values less than 0.05 were considered statistically significant.

\section{Acknowledgments}

We thank Aaron Rae (Emory University Department of Pediatrics and Children's Healthcare of Atlanta Flow Core) for cell sorting. This work was supported by NIH grants (AA01787001, AI083554 to T.L. Denning; DK055679 to A. Nusrat; DK064730 to I.R. Williams; DK72654 and DK79392 to C.A. Parkos), a Career Development Award from the Crohn's and Colitis Foundation of America, and an Emory Egleston Children's Research Center seed grant to T.L. Denning.

Received for publication May 24, 2011, and accepted in revised form September 21, 2011.

Address correspondence to: Timothy L. Denning, Emory University School of Medicine, Whitehead Biomedical Research Building, Room 105R, 615 Michael St., Atlanta, Georgia 30322, USA. Phone: 404.712. 8893; Fax: 404.727.8538; E-mail: tdennin@emory.edu. 
1. Janeway CA Jr, Medzhitov R. Innate immune recognition. Annu Rev Immunol. 2002;20:197-216.

2. Duchmann R, Kaiser I, Hermann E, Mayet W, Ewe $\mathrm{K}$, Meyer zum Buschenfelde KH. Tolerance exists towards resident intestinal flora but is broken in active inflammatory bowel disease (IBD). Clin Exp Immunol. 1995;102(3):448-455.

3. Izcue A, Coombes JL, Powrie F. Regulatory lymphocytes and intestinal inflammation. Annu Rev Immunol. 2009;27:313-338.

4. Macdonald TT, Monteleone I, Fantini MC, Monteleone G. Regulation of homeostasis and inflammation in the intestine. Gastroenterology. 2011; 140(6):1768-1775.

5. Abraham C, Medzhitov R. Interactions between the host innate immune system and microbes in inflammatory bowel disease. Gastroenterology. 2011; 140(6):1729-1737.

6. Jang MH, et al. CCR7 is critically important for migration of dendritic cells in intestinal lamina propria to mesenteric lymph nodes. J Immunol. 2006; 176(2):803-810.

7. Iwata M, Hirakiyama A, Eshima Y, Kagechika H, Kato C, Song SY. Retinoic acid imprints gut-homing specificity on T cells. Immunity. 2004;21(4):527-538.

8. Mora JR, et al. Generation of gut-homing IgAsecreting B cells by intestinal dendritic cells. Science. 2006;314(5802):1157-1160.

9. Mucida D, et al. Reciprocal TH17 and regulatory $\mathrm{T}$ cell differentiation mediated by retinoic acid. Science. 2007;317(5835):256-260.

10. Coombes JL, et al. A functionally specialized population of mucosal CD103+ DCs induces Foxp3+ regulatory $\mathrm{T}$ cells via a TGF-beta and retinoic acid-dependent mechanism. J Exp Med. 2007; 204(8):1757-1764.

11. Sun CM, et al. Small intestine lamina propria dendritic cells promote de novo generation of Foxp3 T reg cells via retinoic acid. J Exp Med. 2007; 204(8):1775-1785.

12. Niess JH, et al. CX3CR1-mediated dendritic cell access to the intestinal lumen and bacterial clearance. Science. 2005;307(5707):254-258.

13. Harrison JK, et al. Role for neuronally derived fractalkine in mediating interactions between neurons and CX3CR1-expressing microglia. Proc Natl Acad Sci U S A. 1998;95(18):10896-10901.

14. Rescigno M. Intestinal dendritic cells. Adv Immunol. 2010;107:109-138.

15. Denning TL, Wang YC, Patel SR, Williams IR, Pulendran B. Lamina propria macrophages and dendritic cells differentially induce regulatory and interleukin 17-producing T cell responses. Nat Immunol. 2007; 8(10):1086-1094.

16. Schulz O, et al. Intestinal CD103+, but not CX3CR1+, antigen sampling cells migrate in lymph and serve classical dendritic cell functions. J Exp Med. 2009;206(13):3101-3114.

17. Pabst O, Bernhardt G. The puzzle of intestinal lamina propria dendritic cells and macrophages. Eur J Immunol. 2010;40(8):2107-2111.

18. Denning TL, et al. Functional specializations of intestinal dendritic cell and macrophage subsets that control Th17 and regulatory $\mathrm{T}$ cell responses are dependent on the T cell/APC ratio, source of mouse strain, and regional localization. J Immunol. 2011;187(2):733-747.

19. Vallon-Eberhard A, Landsman L, Yogev N, Verrier B, Jung S. Transepithelial pathogen uptake into the small intestinal lamina propria. J Immunol. 2006;176(4):2465-2469.

20. Chieppa M, Rescigno M, Huang AY, Germain RN. Dynamic imaging of dendritic cell extension into the small bowel lumen in response to epithelial cell TLR engagement. J Exp Med. 2006;203(13):2841-2852.

21. Niess JH, Adler G. Enteric flora expands gut lamina propria CX3CR1+ dendritic cells supporting inflammatory immune responses under normal and inflammatory conditions. J Immunol. 2010;184(4):2026-2037.

22. Combadiere C, et al. Decreased atherosclerotic lesion formation in CX3CR1/apolipoprotein E double knockout mice. Circulation. 2003;107(7):1009-1016.

23. Smythies LE, et al. Human intestinal macrophages display profound inflammatory anergy despite avid phagocytic and bacteriocidal activity. J Clin Invest. 2005;115(1):66-75.

24. Rescigno $M$, et al. Dendritic cells express tight junction proteins and penetrate gut epithelial monolayers to sample bacteria. Nat Immunol. 2001; 2(4):361-367

25. Qualls JE, Kaplan AM, van Rooijen N, Cohen DA. Suppression of experimental colitis by intestinal mononuclear phagocytes. J Leukoc Biol. 2006; 80(4):802-815.

26. Hunter MM, et al. In vitro-derived alternatively activated macrophages reduce colonic inflammation in mice. Gastroenterology. 2010;138(4):1395-1405.

27. Muehlhoefer A, et al. Fractalkine is an epithelial and endothelial cell-derived chemoattractant for intraepithelial lymphocytes in the small intestinal mucosa. J Immunol. 2000;164(6):3368-3376.

28. Buonocore $S$, et al. Innate lymphoid cells drive interleukin-23-dependent innate intestinal pathology. Nature. 2010;464(7293):1371-1375.

29. Varol C, et al. Monocytes give rise to mucosal, but not splenic, conventional dendritic cells. J Exp Med. 2007;204(1):171-180.

30. Varol C, et al. Intestinal lamina propria dendritic cell subsets have different origin and functions. Immunity. 2009;31(3):502-512.

31. Bogunovic $\mathrm{M}$, et al. Origin of the lamina propria dendritic cell network. Immunity. 2009;31(3):513-525.

32. Gordon S, Taylor PR. Monocyte and macrophage heterogeneity. Nat Rev Immunol. 2005;5(12):953-964.

33. Nakache M, Berg EL, Streeter PR, Butcher EC. The mucosal vascular addressin is a tissue-specific endothelial cell adhesion molecule for circulating lymphocytes. Nature. 1989;337(6203):179-181.

34. Zabel BA, et al. Human G protein-coupled receptor GPR-9-6/CC chemokine receptor 9 is selectively expressed on intestinal homing $\mathrm{T}$ lymphocytes, mucosal lymphocytes, and thymocytes and is required for thymus-expressed chemokine-mediated chemotaxis. J Exp Med. 1999;190(9):1241-1256.

35. Mora JR. Homing imprinting and immunomodulation in the gut: role of dendritic cells and retinoids. Inflamm Bowel Dis. 2008;14(2):275-289.

36. Platt AM, Bain CC, Bordon Y, Sester DP, Mowat AM. An independent subset of TLR expressing CCR2dependent macrophages promotes colonic inflammation. J Immunol. 2010;184(12):6843-6854.

37. Weber B, Saurer L, Schenk M, Dickgreber N, Mueller C. CX3CR1 defines functionally distinct intestinal mononuclear phagocyte subsets which maintain their respective functions during homeostatic and inflammatory conditions. Eur Immunol. 2011;41(3):773-779.

38. Hadis $U$, et al. Intestinal tolerance requires gut homing and expansion of FoxP3+ regulatory T cells in the lamina propria. Immunity. 2011;34(2):237-246.

39. Kamada N, et al. Abnormally differentiated subsets of intestinal macrophage play a key role in Th1-dominant chronic colitis through excess production of IL-12 and IL-23 in response to bacteria. J Immunol. 2005;175(10):6900-6908.

40. Murai M, et al. Interleukin 10 acts on regulatory $\mathrm{T}$ cells to maintain expression of the transcription factor Foxp3 and suppressive function in mice with colitis. Nat Immunol. 2009;10(11):1178-1184.

41. Laukoetter MG, et al. JAM-A regulates permeability and inflammation in the intestine in vivo. J Exp Med. 2007;204(13):3067-3076.

42. Kostadinova FI, Baba T, Ishida Y, Kondo T, Popivanova BK, Mukaida N. Crucial involvement of the CX3CR1-CX3CL1 axis in dextran sulfate sodium-mediated acute colitis in mice. J Lenkoc Biol. 2010;88(1):133-143.

43. Pull SL, Doherty JM, Mills JC, Gordon JI, Stappenbeck TS. Activated macrophages are an adaptive element of the colonic epithelial progenitor niche necessary for regenerative responses to injury. Proc Natl Acad Sci U S A. 2005;102(1):99-104.

44. Ogawa A, Andoh A, Araki Y, Bamba T, Fujiyama Y. Neutralization of interleukin-17 aggravates dextran sulfate sodium-induced colitis in mice. Clin Immunol. 2004;110(1):55-62.

45. Zhang Z, Zheng M, Bindas J, Schwarzenberger P, Kolls JK. Critical role of IL-17 receptor signaling in acute TNBS-induced colitis. Inflamm Bowel Dis. 2006;12(5):382-388.

46. O'Connor W Jr, et al. A protective function for interleukin 17A in T cell-mediated intestinal inflammation. Nat Immunol. 2009;10(6):603-609.

47. Cook DN, et al. Generation and analysis of mice lacking the chemokine fractalkine. Mol Cell Biol. 2001;21(9):3159-3165. 